\title{
The ellipticities of globular clusters in the Andromeda galaxy
}

\author{
A. Staneva ${ }^{1}$, N. Spassova ${ }^{2}$ and V. Golev ${ }^{1}$ \\ 1 Department of Astronomy, Faculty of Physics, St. Kliment Okhridski University of Sofia, 5 James Bourchier street, \\ BG-1126 Sofia, Bulgaria \\ 2 Institute of Astronomy, Bulgarian Academy of Sciences, 72 Tsarigradsko chaussée, BG-1784 Sofia, Bulgaria
}

Received March 15; accepted October 4, 1995

\begin{abstract}
The projected ellipticities and orientations of 173 globular clusters in the Andromeda galaxy have been determined by using isodensity contours and 2D Gaussian fitting techniques. A number of B plates taken with the $2 \mathrm{~m}$ Ritchey-Chretien-coudé reflector of the Bulgarian National Astronomical Observatory were digitized and processed for each cluster. The derived ellipticities and orientations are presented in the form of a catalogue ${ }^{\star}$. The projected ellipticities of M 31 GCs lie between $0.03 \div 0.24$ with mean value $\bar{\varepsilon}=0.086 \pm 0.038$. It may be concluded that the most globular clusters in the Andromeda galaxy are quite spherical. The derived orientations do not show a preference with respect to the center of M31. Some correlations of the ellipticity with other clusters parameters are discussed. The ellipticities determined in this work are compared with those in other Local Group galaxies.
\end{abstract}

Key words: globular clusters: general — galaxies: individual: M 31 — galaxies: star clusters — catalogs

\section{Introduction}

Representing the oldest of all stellar populations, the globular clusters (from now on GCs) are of interest not only for their sake, but also for their bearing on the earliest phases of the galactic evolution. Being dependent upon the galactic tidal field, the initial conditions at the time of their formation, their rotation and velocity anisotropies of cluster members, the shape of GCs can provide insights into their dynamics. The study of GC's flattening can tell us how the globular clusters are formed and how they evolve dynamically.

Ever since the work of Pease \& Shapley (1917) we have known that most GCs in our Galaxy are somewhat flattened. The aspherical appearance of the globulars was first discussed in some details by Shapley (1930), who analyzed the shapes of the clusters by star counts technique. Since then several studies have been published, where the ellipticities of large GC samples in our Galaxy and in nearby galaxies are reported (Kadla et al.1976, 1977; Frenk \& Fall 1982; White \& Shawl 1987).

One of the most striking differences between the GCsystems in our Galaxy and the Magellanic Clouds is that many luminous clusters in the MC are highly flattened. The numerous observations, for instance Geisler \& Hodge (1980), Frenk \& Fall (1982), Geyer et al. (1983), van den

Send offprint requests to: A. Staneva

${ }^{\star}$ Table 1, 2 and 3 are also available in electronic form at the CDS via anonymous ftp 130.79.128.5
Bergh \& Morbey (1984), and Kontizas et al. (1989), have shown that the globulars in LMC are markedly more elliptical than those in our Galaxy. Similarly, Kontizas et al. $(1985,1990)$, have reported that the GCs in SMC are statistically even more flattened than the globulars in LMC.

Although the physical cause of these shape differences is not yet clear, van den Bergh \& Morbey (1984) have suggested that the sequence Galaxy - LMC - SMC represents meanwhile a sequence of decreasing metallicity and increasing flattening. Other possible explanation is suggested by Geisler \& Hodge (1980) for LMC clusters and may be applied here. They have assumed that the gravothermal shock heating, which occurs during each passage of the globular cluster through the galactic disk and that may evaporate the outer clusters stars (this results in decreasing the clusters ellipticity), may be much less significant in the less massive galaxies. The Galaxy LMC - SMC ordering is also the sequence of decreasing masses. Then, under the above assumption, the flattening of the GCs should arise along this sequence. Therefore, it is reasonable to ask where is the place of M 31 clusters in this sequence.

Several studies reporting the shapes of the M 31 GCs appeared in the last years. Pritchet \& van den Bergh (1984) measured the ellipticity of Mayall II, the brightest globular cluster in M31. Lupton (1989, hereafter Lu89) has published the ellipticities of a small sample of 18 clusters in M 31 and Davoust \& Prugniel (1990, hereafter 
DP90) have reported the flattening of 13 of the brightest globulars in M 31 .

Ten years ago with the $2 \mathrm{~m} \mathrm{f} / 8$ Ritchey-Chretien-coudé (2 m RCC) reflector of the Bulgarian National Astronomical Observatory (BNAO), a long-term program for studying the shapes of GCs in Andromeda galaxy was started. This program begun before the availability of high-quality CCD detectors at BNAO. First, the shapes of 5 M 31 globulars have been obtained by photographic isodensitometry processing (Spassova \& Staneva 1984). Next, the ellipticities and orientations of $30 \mathrm{M} 31$ globular clusters, based on isodensity contour analysis of the digitized GC images, have been reported (Staneva et al. 1985). Then we have extended the sample of the processed GCs up to 88 (Spassova et al. 1988).

This paper is a report on the latest step of our program. Here the results of the analysis of the projected ellipticities, $\varepsilon \pm \delta \varepsilon$, and the orientations, $\theta \pm \delta \theta$, of a large sample of 173 brighter $(B \leq 18.5)$ globular clusters in the Andromeda galaxy are presented. This is a total sample of all M 31 globulars ever processed so far by us. This work could be useful as a basis for future morphological analysis of the full GC system in the Andromeda galaxy, which was substantially extended recently (see, for instance, Battistini et al. 1993).

Section 2 describes the observational material and the reduction techniques together with the error analysis. The catalogue with derived parameters is presented in Sect. 3. In Sect. 4, our results are discussed and compared with those of other authors. The ellipticities of M 31 GCs, derived by us, are compared with known ones in other Local Group galaxies. Finally, in Sect. 5 some correlations with other cluster parameters are investigated.

\section{Observations and data reduction}

\subsection{The observational material}

The observational basis of our study was a sample of photographic plates of M 31, covering the whole galaxy. We used 7 B-plates (Kodak IIaO and ORWO ZU21 emulsions, WK 38 glass filter), exposed between 60 and 90 min, taken on the $2 \mathrm{~m} \mathrm{RCC} f / 8$ reflector of the BNAO (plate scale 12.89 arcsec $\mathrm{mm}^{-1}$, mean seeing about $2^{\prime \prime}$ ). In addition, 2 B-plates taken with the $1.82 \mathrm{~m}$ telescope at Asiago Observatory on $103 \mathrm{aO}$ emulsion with GG 13 glass filter (plate scale $12.59 \operatorname{arcsec} \mathrm{mm}^{-1}$ ) were kindly provided to us by colleagues of the Asiago Observatory. 173 globular clusters were selected from the lists of cluster candidates published by "M 31 consortium" (Sargent et al. 1977) and by "Bo-Group" from Bologna University (Battistini et al. 1980, 1987, 1993). This sample includes almost all clusters brighter than $B=18^{\mathrm{m}} .5$ that are located in the main body of the Andromeda galaxy.

All plates were digitized and the density distributions were obtained using the MDM-6 Joyce-Loebl microdensit- ometer of BNAO. A 12-bit digitization was taken with 40 $\mu \mathrm{m}$ squared spot size and $25 \mu \mathrm{m}$ increment size and for each object a $64 \times 64$ raster frames of blackening density, $D_{i j}$, were formed. These 2D frames were used to estimate the flattening of the cluster images expressed in terms of ellipticities, $\varepsilon=1-b / a$, where $a$ and $b$ are the projected major and minor semiaxes, and the orientation, $\theta$, is expressed as position angles measured eastward from north. On each plate at least 10 star images were also digitized to estimate the shape of the point-spread functions (PSFs) at the time the observations were made. Being slightly noncircular, these images account for the atmospheric dispersion and the tracking/guiding errors. The mean FWHM of stellar images over all plates used by us is $\approx 1^{\prime \prime} .9$ ranging from $1^{\prime \prime} .3$ for the best plate to $2^{\prime \prime} .5$ for the worst one.

\subsection{Processing the density distributions}

We have been engaged for a long time in the development of a reduction, PC-oriented software for the purposes of our study. We had begun this project before the other general-purpose packages were generally available, these, in particular, are not yet available in suitable form in Bulgaria. Thus we chose to go on developing our software with the goal of finishing our investigation of M 31 GCs in a uniform way.

A variety of approaches to extract the morphological data such as flattening, $\varepsilon$, and orientation, $\theta$, from $2 \mathrm{D}$ frames like ours is possible. For clusters lying in uncrowded areas, where the M31 stellar background contributes in simple form, straightforward processing procedures are quite effective. These are limited only by the requirements that it is possible to model the M 31 background in some suitable fashion and that there are no neighbouring stars close to the image of interest. We used both the isodensity analysis by ellipse fits and 2D Gaussian fits to each image array $D(x, y)$.

\subsubsection{Finding the sky level}

In general, the mean sky level around each cluster was determined as the mode of the distribution of pixel values. The mode as a merit of the mean sky level is the most appropriate sky estimator in crowded field situations (see, e.g., Janes \& Heasley 1993) like ours where the clusters are projected on the nonuniform stellar background of M31. The question about the relative merits of using the mode of the sky histogram as the best representation of the background has been often discussed, as it can be seen in Da Costa (1992), Eaton (1989), and Stetson (1987)

However, the nonuniformity of the sky background surrounding M 31 clusters, especially when the clusters are close to spiral arms, does not allow good estimations only by means of the mode of the sky histogram. In these cases there is a significant gradient in the sky across the field 
that makes it difficult to find the peak of the sky histogram. We decided it could be more advantageous to include the sky background directly in the 2D Gaussian least-squares fits.

\subsubsection{Isodensity contours analysis}

The ellipse fits of isolevel sections of astronomical images are a well known tool to estimate ellipticities and position angles of these images (Pasian et al. 1989). Quasicontinuous objects like distant M31 globulars (crowded beyond the spatial resolution limit) can be usefully explored by standard least-squares fits of an ellipse to a series of selected isodensity contours. A direct approach to ellipse fitting is described by Bendinelli et al. (1986) where the usual difficulties in fitting five nonlinear parameters are overcome by applying a Newton-Gauss method improved by Tikhonov's regularization (see for details Bendinelli et al. 1987).

If we define a coordinate system $(x, y)$ consistent with the scanned area, then the system $\left(x^{\prime}, y^{\prime}\right)$ rotated by angle $\theta$ and translated at the ellipse center $x_{\mathrm{c}}, y_{\mathrm{c}}$ with respect to $(x, y)$ allows to describe the ellipse by the canonical equation

$$
(1-\varepsilon)^{2} x^{\prime 2}+y^{\prime 2}=(1-\varepsilon)^{2} a^{2}
$$

Here

$$
\begin{aligned}
& x^{\prime}=\left(x-x_{\mathrm{c}}\right) \cos \theta+\left(y-y_{\mathrm{c}}\right) \sin \theta \\
& y^{\prime}=-\left(x-x_{\mathrm{c}}\right) \sin \theta+\left(y-y_{\mathrm{c}}\right) \cos \theta
\end{aligned}
$$

After appropriate substitutions, a nonlinear equation $f\left(x, y, c_{k}\right)=0$ with respect to the coefficients $c_{k}, k=$ $1, \ldots, 5$, is solved in order to estimate the values of $\varepsilon$ and $\theta$.

\subsubsection{Two-dimensional Gaussian approximation}

In principle, a better way to approximate the blackening density distribution is to use the 2D Gaussian fitting function with well known physical background (cf. Kroll \& Neugebauer 1993). Passing through the atmosphere, the light from the stars will be diffracted, scattered and absorbed. Passing then the telescope, the light distribution of the point-like sources like stars is transformed by the PSF of the telescope. Finally, the star light is scattered and absorbed once again (and some diffusion processes also take place further) when the photographic emulsion is reached. As a result, a (nearly) 2D Gaussian distribution of the blackening density, $D(x, y)$, is recorded on the plate. All this philosophy remains valid for the images of the M 31 globulars, because in general they are quite starlike with a typical FWHM, in our case just 3 or 4 times larger than the FWHM of the PSF.
The density, $D(x, y)$, as a function of position and shape for unsaturated photographic cluster image is well represented by the 2D Gaussian. We chose to use the generalization of this function published by Sanders \& Schröder (1980) especially for the case of the photographic detector. Thus

$I \sim D(x, y)=D_{\text {sky }}(x, y)+a_{3} \mathrm{e}^{-\frac{1}{2} \phi\left(x, y, a_{k}\right)}$

where $I$ represents the falling light intensity, $D_{\text {sky }}(x, y)=$ $a_{0}+a_{1} x+a_{2} y$ describes a plane sky background, $a_{3}$ allows for the central density above the sky level, and $k=4, \ldots, 8$. Here

$\phi\left(x, y, a_{k}\right)=\frac{1}{1-a_{8}}$

$\left(\left(\frac{x-a_{4}}{a_{5}}\right)^{2}-2 a_{8}\left(\frac{x-a_{4}}{a_{5}}\right)\left(\frac{y-a_{6}}{a_{7}}\right)+\left(\frac{y-a_{6}}{a_{7}}\right)^{2}\right)$

where $a_{4}=x_{\mathrm{c}}$ and $a_{6}=y_{\mathrm{c}}$ describe the coordinates of the photometric center of the clusters image, $a_{5}=\sigma_{x}$ and $a_{7}=\sigma_{y}$ are the measures of the image size in $x$ and $y$ directions $(\mathrm{FWHM}=2.355 \sigma)$, and $a_{8}=\rho$ is the correlation coefficient. Coefficients $a_{5}, a_{7}$ and $a_{8}$ describe the overall ellipticity of the image with arbitrary orientation and axis size.

To make some photometry with this generalized Gaussian distribution we need to reconstruct the intensity, $I$, based on the knowledge of the accurate characteristic curves. However, to extract the morphological parameters like ellipticity and its orientation, it is not necessary to apply such reconstruction. The reason is that only the intensity of the falling light of the source, but not its positional distribution (which is left unchanged on the plate), is distorted by the characteristic curve.

\subsection{Error analysis}

\subsubsection{The influence of the signal-to-noise ratio}

It is important to analyze what is the influence of the observational Signal-to-Noise Ratio (SNR) on the shape and orientation of the images of the M 31 globulars. It may be assumed that a given cluster is intrinsically spherical in shape but its observations have errors resulting from the noise. Then the cluster will, on average, look slightly elongated. The fainter the cluster is, the more important the artificial elongation of the cluster image due to the observational SNR will be. As a result one would expect observational errors to make the faint clusters seem flatter and more uncertain in orientation than luminous ones.

To investigate this we have produced a series of Monte Carlo simulations by constructing artificial samples of M 31 globulars, convolving with real PSF $\left(\mathrm{FWHM} \sim 2^{\prime \prime} .5\right)$ 
measured from our plates and adding different SNR at given model ellipticities $\left(0.00 \leq \varepsilon_{\text {model }} \leq 0.10\right)$.

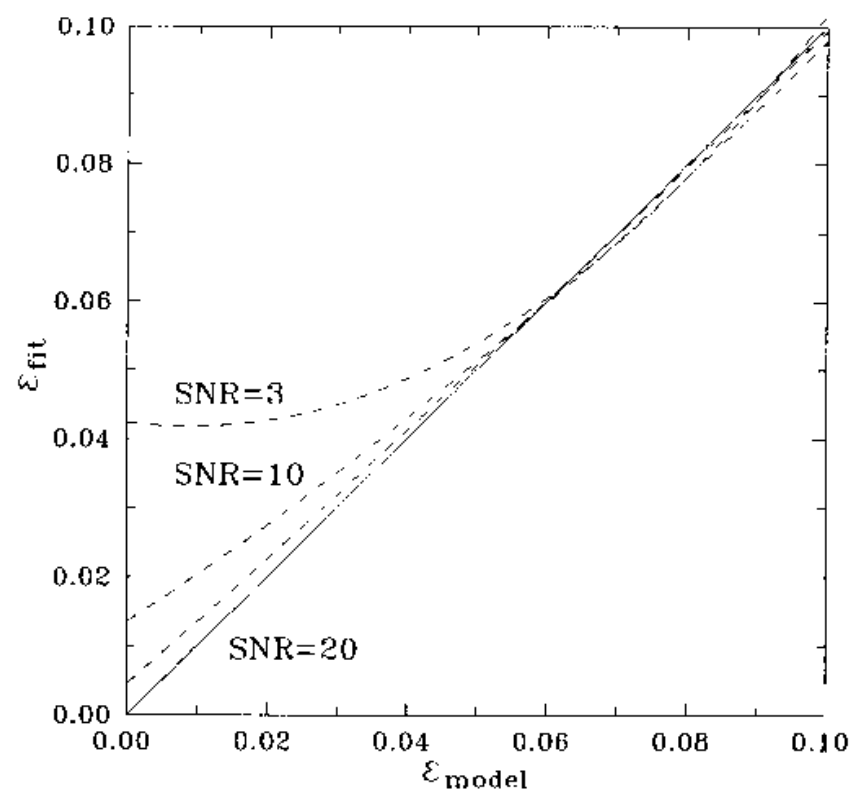

Fig. 1. The influence of the errors induced by SNR of the Monte Carlo simulated ellipticities, $\varepsilon_{\text {model }}$, on the ellipticity values taken from the fit to the model data, $\varepsilon_{\text {fit }}$. Dashed curves represent dependences for $\mathrm{SNR}=3, \mathrm{SNR}=10$ and $\mathrm{SNR}=20$, respectively $\left(45^{\circ}\right.$ theoretical line is also shown)

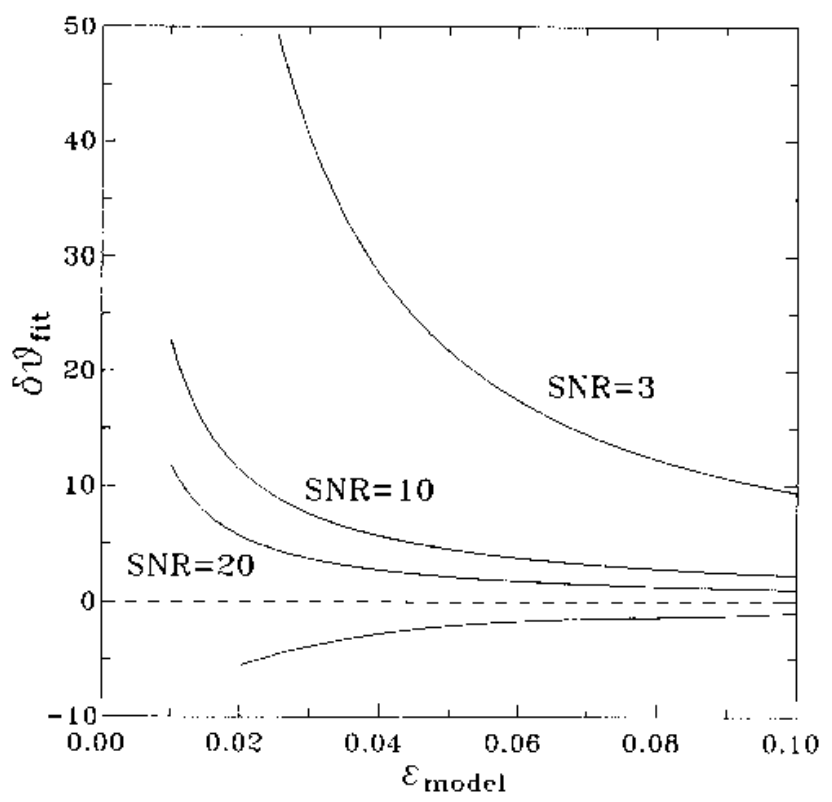

Fig. 2. The orientation error, $\delta \theta_{\text {fit }}$, derived by the fit versus the model ellipticity, $\varepsilon_{\text {model }}$, for three values of SNR. The model orientation (plotted with dashed line) is taken to be always $0^{\circ}$

The result is shown in Fig. 1, where the model values of ellipticities, $\varepsilon_{\text {model }}$, and the ellipticity values taken from the fit to the model data, $\varepsilon_{\text {fit }}$, are compared. It is seen that the maximal ellipticity increasing due to the observational noise does not exceed 0.02 at $\mathrm{SNR}=10$ and $\varepsilon_{\text {model }}=0$.

The influence of the SNR on the derived orientations may be estimated in analogous way.

Figure 2 shows the error in orientation $\delta \theta_{\text {fit }}$ (in degrees) derived by the fit versus the model ellipticity with model orientation being always $0^{\circ}$. As it would be expected, the more accentuated is the ellipticity, the smaller is the error in orientation.

In our real sample we have included only clusters with $B \leq 18^{\mathrm{m}}$. That limit is at least $2^{\mathrm{m}}$ above the plate threshold and all the GCs considered here are observed with SNR $\geq 10$, mostly being around SNR $\sim 25$. Thus the noise-induced error in $\varepsilon(\sim 0.02$ in the worst case $)$ and in $\theta$ (typically $\lesssim 5^{\circ}$ at $\varepsilon \approx 0.02$ and $10^{\circ}$ at the worst case) does not affect the shape parameters in any substantial way.

\subsubsection{Fit problems and fitting errors}

Basically there is no problem to achieve 2D Gaussian fits in the Newton-Raphson scheme providing the appropriate initial values that allow a convergence. On the average, we needed only 3 or 4 iterations to reach the common $\chi^{2}$ convergence criterion. The formal errors in the leastsquares $2 \mathrm{D}$ Gaussian fit are, typically, \pm 0.005 in $\varepsilon$ and $\pm\left(2^{\circ} \div 5^{\circ}\right)$ in $\theta$. Even the faintest clusters with lowest SNR do not cause convergence problems.

However, it is not so when the isodensity contours analysis is used and least-squares ellipse fits are applied. A detailed empirical analysis of the errors of the least-squares ellipse fits versus the radial SNR is presented in Pasian et al. (1989). The main conclusion is that such fits have to be preferred only if the shape of the density contours can be well described by smooth enough isolines. In our case the typical formal error of the least-squares ellipse solutions are \pm 0.01 in $\varepsilon$ and $\pm 5^{\circ}$ in $\theta$.

In case of spatially resolved morphological details like faint background stars and emulsion grains outlined by the lowest isolines, the ellipse fits should be used with great precautions. When the radial SNR becomes low as in the cases of the outermost contours passing at densities only of $0.1-0.2$ above background, the fitted ellipses yield bias errors that grow rapidly with the radial noise caused by the emulsion grains.

We have used data derived by both methods, thus inducing an additional error due to their combination. In Fig. 3 we have shown the ellipticities, determined by isodensity contour analysis versus the ellipticities, determined by 2D Gaussian fits (Fig. 3a) and the corresponding orientations (Fig. 3b) for the plate No. 356. The corresponding relations are

$\varepsilon_{\text {iso }}=(0.012 \pm 0.004)+(0.901 \pm 0.040) \varepsilon_{\text {Gauss }}$, 

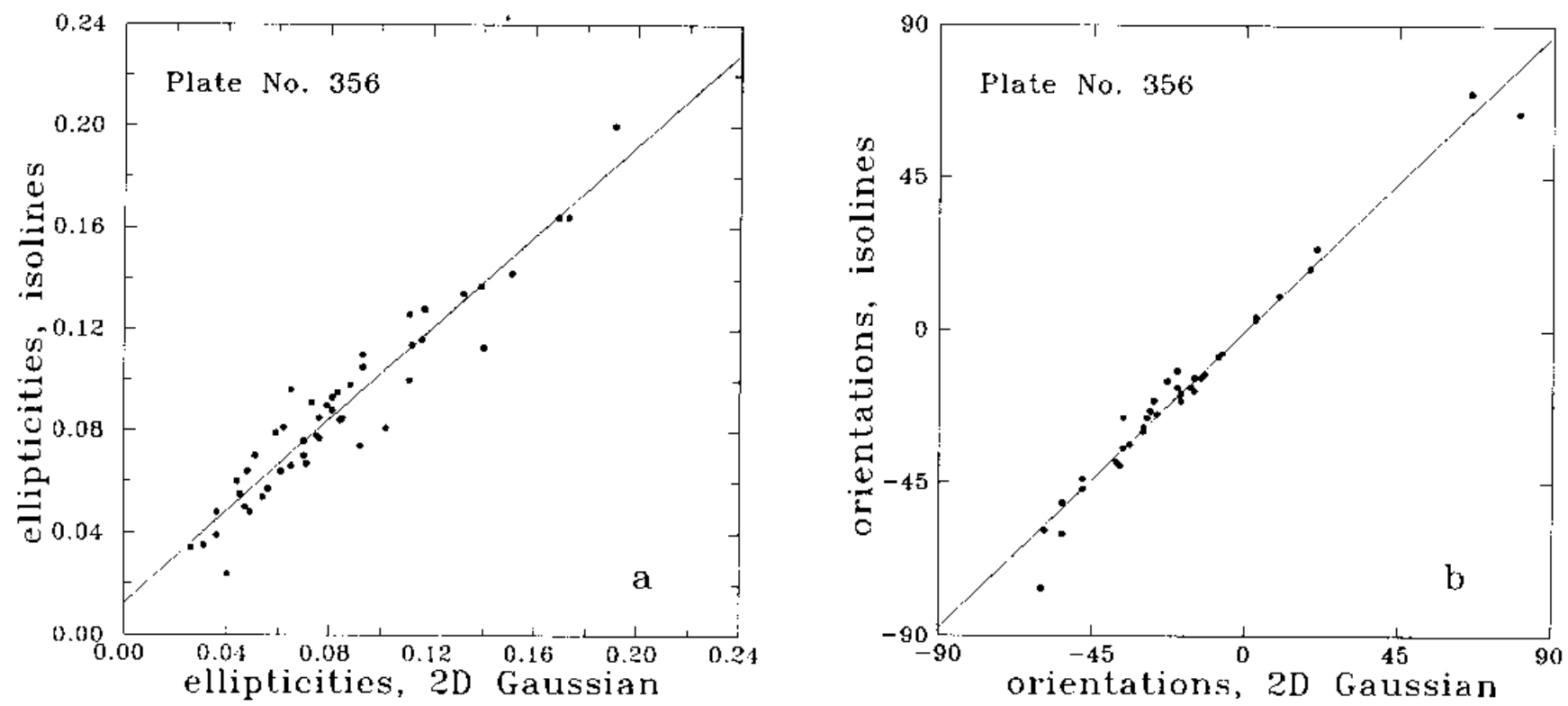

Fig. 3. Plate No. 356: a) ellipticities determined by isodensity contour analysis versus the ellipticities determined by $2 \mathrm{D}$ Gaussian fits, b) the corresponding orientations

$\theta_{\text {iso }}=(-0.161 \pm 0.984)+(0.973 \pm 0.028) \theta_{\text {Gauss }}$,

where the correlation coefficients are 0.915 and 0.971 , respectively. The corresponding fit standard errors induced by combination of the two methods are $\sim 0.01$ in $\varepsilon$ and $\sim 5^{\circ}$ in $\theta$.

It can be seen that the results derived by both methods are in satisfactory agreement as long as the radial SNR is high enough, as it is usual in most cases.

\subsubsection{Interplate comparison}

To figure out shape parameters of each cluster we have used up to 5 different plates to minimize the nonlinearity of plate response. To check the influence of the different seeing conditions we have examined the repeatibility of the ellipticities (Fig. 4) and the orientations determined by both fitting methods on plates No. 356 (the best one FWHM $\sim 1^{\prime \prime} .5$ ) and plate No. 665 (the worst one - FWHM $\sim 2$ '. 8 ). The corresponding relations are

$\varepsilon_{\mathrm{pl} .665}=(0.026 \pm 0.007)+(0.734 \pm 0.084) \varepsilon_{\mathrm{pl} .356}$

$\theta_{\mathrm{pl} .665}=(-1.047 \pm 3.541)+(1.081 \pm 0.104) \theta_{\mathrm{pl} .356}$

where the correlation coefficients are 0.669 and 0.817 , respectively. The corresponding fit standard errors are $\sim 0.015$ in $\varepsilon$ and $\sim 15^{\circ}$ in $\theta$.

We have made an analysis of the distribution of the ellipticities for the clusters at widely different distances from the center of the plates. The ellipticities show no

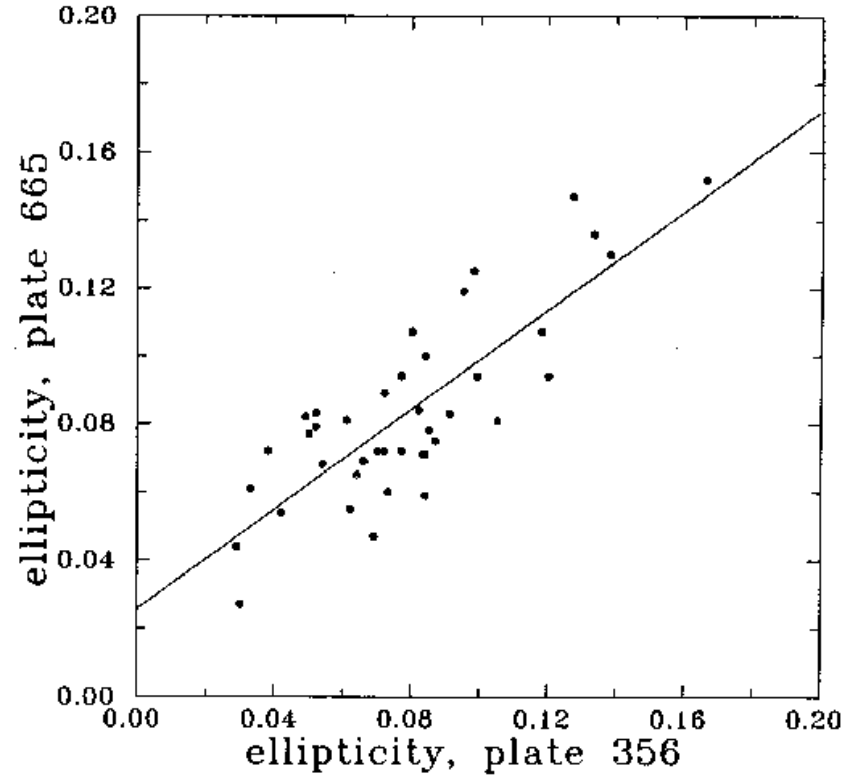

Fig. 4. Repeatibility of the ellipticities on plates No. 356 and No. 665

significant systematic differences as a function of distance from the plate center. The same, also, is valid for orientations.

\subsubsection{Final errors}

The above findings suggest that different sources of errors do not add significant systematic contributions. The 

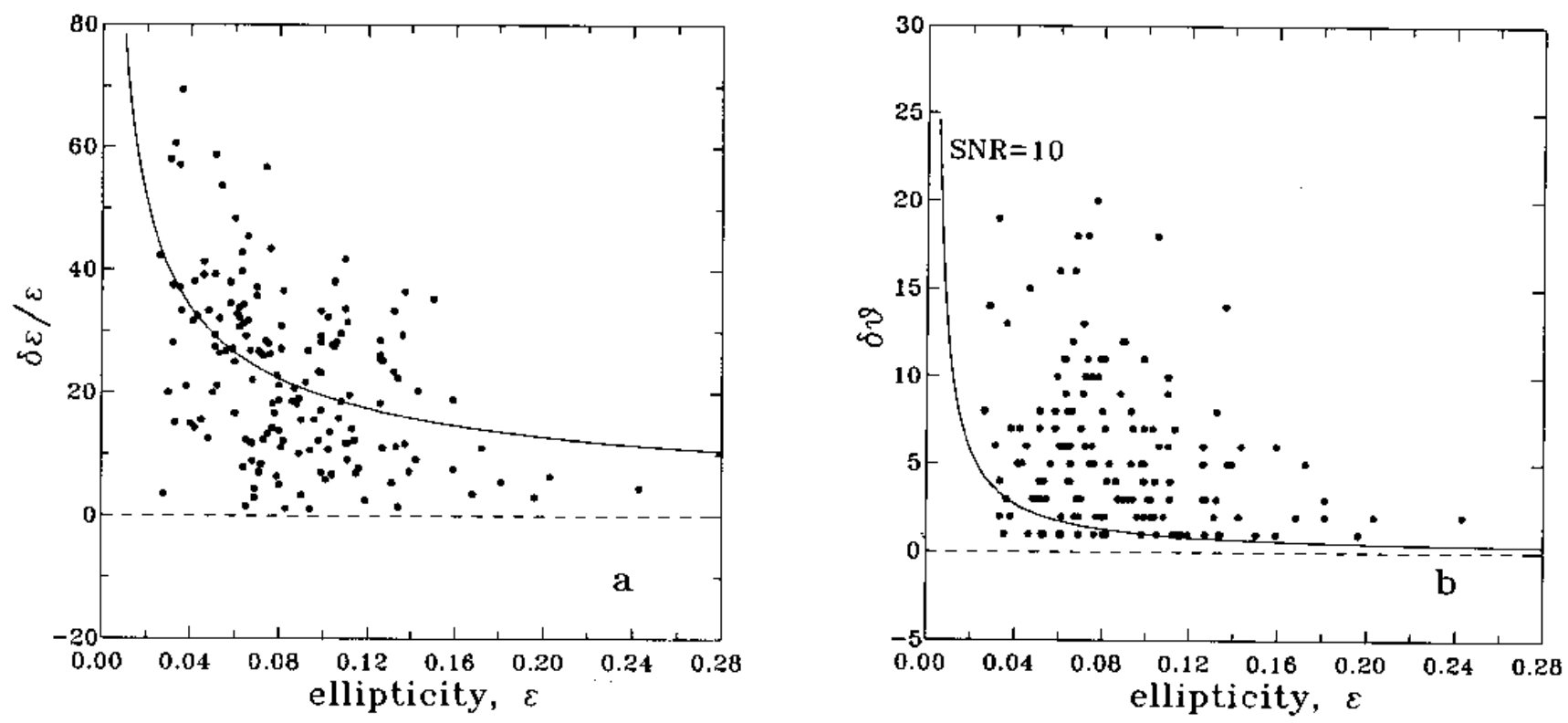

Fig. 5. Final errors: a) relative ellipticity errors $\delta \varepsilon / \varepsilon$ (in \%) as a function of the derived ellipticities $\varepsilon$ follow a power law $\left.\delta \varepsilon / \varepsilon \propto \varepsilon^{-0.6}, \mathbf{b}\right)$ orientation errors $\delta \theta$ versus the derived ellipticities $\varepsilon$ (SNR $=10$ line from Fig. 2 is overplotted to show the limit of uncertainty due to the fitting procedure)

final errors, $\delta \varepsilon$ and $\delta \theta$, are derived as root mean square of the errors of each individual estimate. Their values are, typically, $\pm(0.020 \div 0.025)$ in $\varepsilon$ and $\pm\left(5^{\circ} \div 20^{\circ}\right)$ in $\theta$, comparable with the rms contribution of sources discussed above. These errors are reported in Table 1 below.

In Fig. 5a the relative ellipticity errors $\delta \varepsilon / \varepsilon$ in percents are plotted versus the derived ellipticities. It can be seen that the typical relative error in the ellipticity follows a power law in form $\delta \varepsilon / \varepsilon \propto \varepsilon^{-0.6}$, reaching a maximal value $\sim 70 \%$ at nearly circular images with $\varepsilon \approx 0.03$.

In Fig. 5b final orientation errors are drawn against the ellipticity. The curve for SNR $=10$ from Fig. 2 is overploted there to show the limit of uncertainty due to our fitting procedure.

The final shape parameters, $\varepsilon$ and $\theta$, are estimated by both methods as mean values over all plates used for each cluster. These derived parameters have not been corrected in any way for seeing effects. The estimations of star images flattening are the best way to give a sense for the influence of the seeing. The mean ellipticity of stellar images over all used plates is $0.070 \pm 0.025$ ranging from 0.03 to 0.12 over the particular plates. It is well known (Lupton 1989) that the flattening of the stellar images is due both to the atmospheric dispersion and to the residual tracking/guiding errors of the telescope. Thus the derived ellipticity values inevitably reflect both their true properties and seeing effects. Following Lupton, in view of the flattened stellar images it is most natural to interpret the measured $\varepsilon$ as an upper limit to the true flattening of the M31 GCs.

\section{The catalogue}

The morphology of M31 GCs described by its shape parameters derived by us are given in Table 1 .

Column 1 contains the name from M31 consortium (Sargent et al. 1977) or from Bo-Group (Battistini et al. 1980, 1987, 1993). Column 2 shows the mean ellipticity, $\bar{\varepsilon}$, with its final rms uncertainty, $\delta \varepsilon$, determined over all plates containing given cluster. Column 3 gives the position angle of the major axis, $\bar{\theta}$, measured from north toward east, together with its final rms uncertainty, $\delta \theta$ (determined also over all plates having this cluster). The number of separate measurements (all plates and all methods) is given in Column 4. Column 5 lists the method of reduction for each cluster (I for isodensity analysis and D for two-dimensional Gaussian fit). Column 6 gives the apparent angular size (in arcsec) of the major axis for the outer isodense contour (at $3 \sigma$-level above background) or the FWHM of the major axis depending on the method used.

The values of the ellipticities given in Table 1 differ for some clusters more than $2.5 \delta \varepsilon$ from the data published previously by us. There are four reasons for this:

1. The incorrect identification as in the case of $G 110$;

2 . The overlap with close faint neighbor image causing a deformation of the outer isodense contours ( $G 64$, $G$ 124, and G 267);

3 . The different quality of plate material used ( $G 76$, $G$ 113, G 212, G 216, G 275, G 282, and G 301);

4. The influence of the stellar background nonuniformity toward the center of M 31 like G 199 . 
The new shape parameters of the above clusters, listed in Table 1, are derived from at least 2 plates using both methods of processing with exception of the data for $G 110$ and $G 199$.

\section{Results and discussion}

\subsection{Overall properties of our sample}

Data catalogued in Table 1 allow us to outline some overall statistical properties of our sample of M31 GCs.

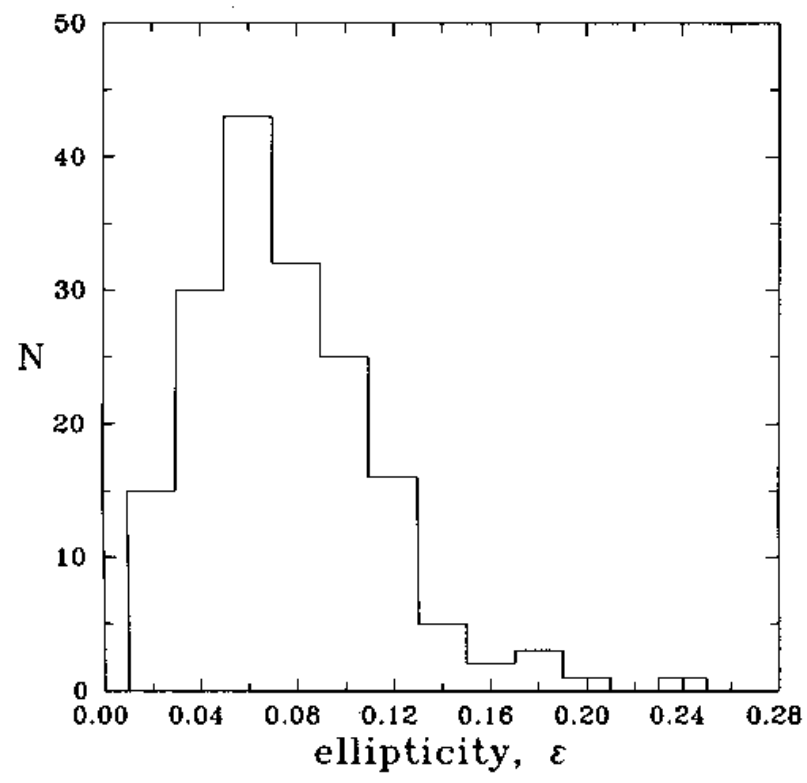

Fig. 6. A histogram of the distribution of the projected ellipticities of 173 GCs in M 31

In Fig. 6 the histogram of the distribution of the projected ellipticities of all examined up to now $173 \mathrm{GCs}$ is shown. These ellipticities lie between $0.03 \div 0.24$ with mean value $\langle\varepsilon\rangle=0.086 \pm 0.038$. The already published ellipticities of M II ( 0.22 in Pritchet \& van den Bergh 1984; 0.20 in Lu89 and DP90), of M III (0.05 in Lu89) and of $G 185$ (0.10 in DP90) lie also in the above range and do not significantly change the mean value. It can be seen that $70 \%$ of GCs have $\varepsilon<0.10$, while only 2 clusters are flattened more than 0.20 . Thus it may be concluded that most of the globulars in the Andromeda galaxy are quite spherical.

Let us compare the mean ellipticity for $173 \mathrm{GCs}$ in M 31 with the mean ellipticities for GCs of four other galaxies studied up to now. The comparative data are presented in Table 2 together with other useful information. Two main conclusions can be drawn from this table:

1. The apparent ellipticities of M31 GCs cover the same range of ellipticities as do the galactic globulars. The GCs in both galaxies are nearly spherical.
2. The flattening of M31 globulars seems to differ markedly from clusters in the LMC and SMC, the latter being highly flattened.

We have computed the cluster ellipticities and orientations as a function of cluster positions in the M 31 plane in relative coordinates as defined by Huchra et al. (1991). There is no trend in the orientations toward the center of M 31, like the situation in our Galaxy (see White \& Shawl 1987). The most convenient interpretation is that the tidal forces of the M31 main body do not cause any significant influence on the orientations of its globulars.

\subsection{Comparison with other authors}

The ellipticities determined in this work have been compared with those from Lu89 and DP90. Lupton has measured the ellipticities of $18 \mathrm{M} 31 \mathrm{GCs}$ in three different annuli, A, B and C, with radii $2^{\prime \prime} .25-4^{\prime \prime} .50,4^{\prime \prime} .50-6^{\prime \prime} .75$, and $5^{\prime \prime} .25-7^{\prime \prime} .20$ respectively. DP90 have determined the clusters ellipticities in two annual areas, $\mathrm{A}$ and $\mathrm{B}$, with radii $2^{\prime \prime} \div 4^{\prime \prime}$ and $4^{\prime \prime} \div 6^{\prime \prime}$ respectively. 16 clusters from our list are in common with Lupton's data and 11 - with Davoust \& Prungniel ones. The present results compared with those of the above mentioned authors are listed in Table 3 where Column 1 gives the cluster name, Columns 2 and 3 contain the ellipticities in annual areas $\mathrm{A}$ and B from Table 1 of DP90 and from Table 2 of Lu89, respectively, Column 4 shows the ellipticities derived in our study, and Column 5 lists the radii of outer isodensity contours in arcsec used by us.

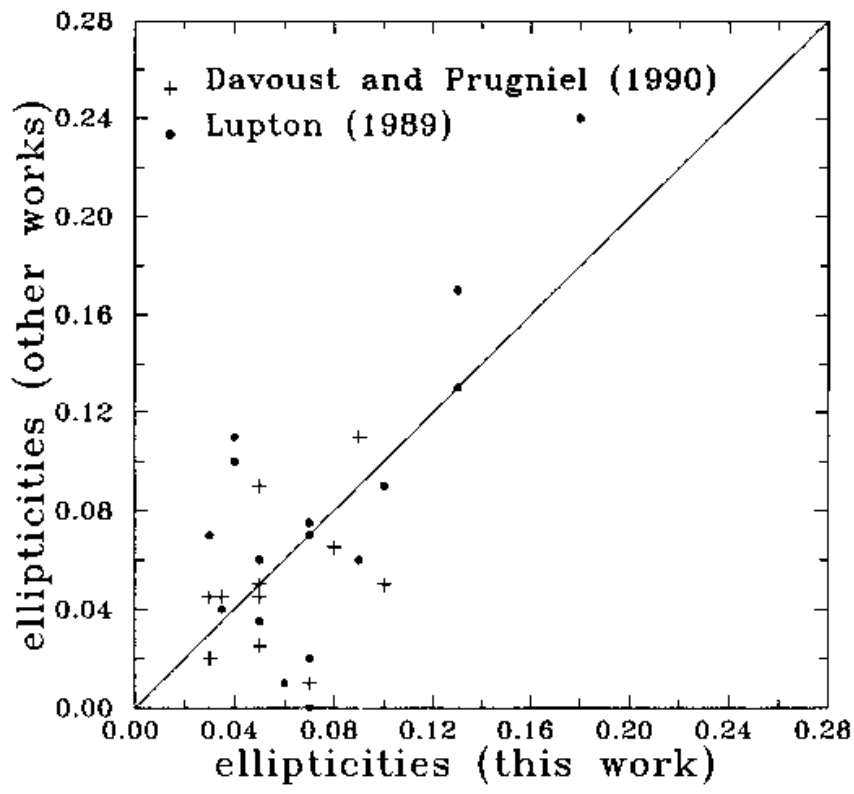

Fig. 7. Our ellipticities versus the ellipticities of Lu87 (filled circles) and DP90 (crosses)

Our estimations of the ellipticities of clusters in common are plotted in Fig. 7 against the values determined by 
Table 1. Morphological parameters of M31 globulars

\begin{tabular}{|c|c|c|c|c|c|c|c|}
\hline \multirow[t]{2}{*}{ name } & \multicolumn{2}{|c|}{ ellipticity } & \multicolumn{2}{|c|}{ P.A. } & \multirow{2}{*}{$\begin{array}{l}\text { number of } \\
\text { measure - } \\
\text { ments }\end{array}$} & \multirow[t]{2}{*}{ method } & \multirow{2}{*}{$\begin{array}{l}\text { apparent } \\
\text { angular size, } \\
\text { arcsec }\end{array}$} \\
\hline & $\bar{\varepsilon}$ & $\delta \varepsilon$ & $\bar{\theta}$ & $\delta \theta$ & & & \\
\hline G014 & 0.126 & 0.032 & 155 & 6 & 2 & $\mathrm{I}, \mathrm{G}$ & 3.7 \\
\hline G022 & 0.127 & 0.032 & 140 & 1 & 2 & $\mathrm{I}, \mathrm{G}$ & 3.0 \\
\hline G029 & 0.159 & 0.030 & 45 & 6 & 1 & I & 2.2 \\
\hline G031 & 0.139 & 0.010 & 169 & 5 & 1 & $\mathrm{G}$ & 1.5 \\
\hline G033 & 0.064 & 0.022 & 143 & 7 & 2 & $\mathrm{I}, \mathrm{G}$ & 3.1 \\
\hline G035 & 0.074 & 0.021 & 95 & 5 & 4 & $\mathrm{I}, \mathrm{G}$ & 4.0 \\
\hline G036 & 0.137 & 0.050 & - & - & 1 & $\mathrm{G}$ & 2.2 \\
\hline G037 & 0.136 & 0.041 & 160 & 14 & 1 & $\mathrm{G}$ & 2.4 \\
\hline G038 & 0.098 & 0.023 & 30 & 5 & 5 & $\mathrm{I}, \mathrm{G}$ & 3.7 \\
\hline G039 & 0.089 & 0.009 & 66 & 3 & 3 & $\mathrm{I}, \mathrm{G}$ & 2.6 \\
\hline G040 & 0.099 & 0.023 & - & - & 4 & $\mathrm{I}, \mathrm{G}$ & 2.8 \\
\hline G041 & 0.028 & 0.001 & 56 & 14 & 2 & $\mathrm{I}, \mathrm{G}$ & 4.7 \\
\hline G042 & 0.131 & 0.007 & 72 & 2 & 2 & $\mathrm{I}, \mathrm{G}$ & 3.3 \\
\hline G044 & 0.243 & 0.011 & 34 & 2 & 4 & $\mathrm{I}, \mathrm{G}$ & 2.0 \\
\hline G049 & 0.172 & 0.019 & 168 & 5 & 2 & $\mathrm{I}, \mathrm{G}$ & 2.3 \\
\hline G052 & 0.067 & 0.018 & 73 & 2 & 6 & $\mathrm{I}, \mathrm{G}$ & 2.8 \\
\hline G053 & 0.114 & 0.014 & 114 & 1 & 4 & $\mathrm{I}, \mathrm{G}$ & 3.5 \\
\hline G058 & 0.069 & 0.002 & 156 & 2 & 4 & $\mathrm{I}, \mathrm{G}$ & 6.5 \\
\hline G060 & 0.108 & 0.020 & 44 & 2 & 3 & $\mathrm{I}, \mathrm{G}$ & 2.5 \\
\hline G061 & 0.033 & 0.001 & 178 & 2 & 1 & $\mathrm{G}$ & 2.6 \\
\hline G062 & 0.051 & 0.015 & 60 & 3 & 5 & $\mathrm{I}, \mathrm{G}$ & 3.4 \\
\hline G063 & 0.038 & 0.008 & 21 & 2 & 2 & $\mathrm{I}, \mathrm{G}$ & 2.2 \\
\hline G064 & 0.035 & 0.020 & 101 & 1 & 2 & $\mathrm{I}, \mathrm{G}$ & 4.7 \\
\hline G065 & 0.051 & 0.021 & - & - & 1 & G & 2.8 \\
\hline G069 & 0.107 & 0.017 & - & - & 2 & $\mathrm{G}$ & 2.1 \\
\hline G070 & 0.058 & 0.020 & - & - & 3 & $\mathrm{I}, \mathrm{G}$ & 3.2 \\
\hline G071 & 0.059 & 0.005 & 26 & 8 & 2 & G & 2.4 \\
\hline G072 & 0.090 & 0.001 & 179 & 2 & 1 & G & 2.0 \\
\hline G073 & 0.053 & 0.017 & 71 & 1 & 2 & $\mathrm{I}, \mathrm{G}$ & 5.0 \\
\hline G076 & 0.032 & 0.009 & 158 & 5 & 5 & $\mathrm{I}, \mathrm{G}$ & 5.2 \\
\hline G077 & 0.031 & 0.018 & 10 & 6 & 2 & $\mathrm{G}$ & 2.1 \\
\hline G078 & 0.077 & 0.009 & 92 & 2 & 6 & $\mathrm{I}, \mathrm{G}$ & 6.5 \\
\hline G081 & 0.143 & 0.029 & 144 & 6 & 2 & $\mathrm{I}, \mathrm{G}$ & 3.3 \\
\hline G084 & 0.111 & 0.014 & 68 & 4 & 1 & G & 2.3 \\
\hline G087 & 0.035 & 0.013 & - & - & 5 & $\mathrm{I}, \mathrm{G}$ & 3.3 \\
\hline G088 & 0.046 & 0.018 & 170 & 1 & 2 & $\mathrm{I}, \mathrm{G}$ & 2.0 \\
\hline G090 & 0.077 & 0.014 & 46 & 20 & 3 & $\mathrm{I}, \mathrm{G}$ & 2.7 \\
\hline G094 & 0.203 & 0.013 & 171 & 2 & 2 & G & 3.1 \\
\hline G096 & 0.042 & 0.016 & 27 & 7 & 3 & $\mathrm{I}, \mathrm{G}$ & 4.5 \\
\hline G098 & 0.069 & 0.003 & 70 & 1 & 2 & $\mathrm{I}, \mathrm{G}$ & 2.5 \\
\hline G101 & 0.084 & 0.025 & - & - & 1 & $\mathrm{G}$ & 2.0 \\
\hline G102 & 0.098 & 0.012 & 60 & 1 & 4 & $\mathrm{I}, \mathrm{G}$ & 2.1 \\
\hline G104 & 0.082 & 0.010 & 56 & 1 & 3 & $\mathrm{I}, \mathrm{G}$ & 2.4 \\
\hline G105 & 0.090 & 0.003 & 160 & 3 & 2 & $\mathrm{I}, \mathrm{G}$ & 3.8 \\
\hline G106 & 0.127 & 0.006 & - & - & 2 & $\mathrm{I}, \mathrm{G}$ & 2.5 \\
\hline G107 & 0.061 & 0.020 & 65 & 1 & 3 & $\mathrm{I}, \mathrm{G}$ & 2.0 \\
\hline G108 & 0.036 & 0.012 & 33 & 13 & 4 & $\mathrm{I}, \mathrm{G}$ & 3.5 \\
\hline G110 & 0.063 & 0.001 & 32 & 4 & 2 & G & 2.3 \\
\hline G112 & 0.137 & 0.016 & 167 & 5 & 4 & $\mathrm{I}, \mathrm{G}$ & 2.2 \\
\hline G113 & 0.032 & 0.012 & 115 & 19 & 4 & $\mathrm{I}, \mathrm{G}$ & 2.5 \\
\hline G114 & 0.102 & 0.033 & 80 & 2 & 6 & $\mathrm{I}, \mathrm{G}$ & 2.8 \\
\hline G116 & 0.113 & 0.016 & 61 & 7 & 4 & $\mathrm{I}, \mathrm{G}$ & 2.5 \\
\hline G117 & 0.105 & 0.047 & 44 & 18 & 1 & I & 1.8 \\
\hline G119 & 0.050 & 0.010 & 40 & 3 & 5 & $\mathrm{I}, \mathrm{G}$ & 5.2 \\
\hline
\end{tabular}


Table 1. continued

\begin{tabular}{|c|c|c|c|c|c|c|c|}
\hline \multirow[t]{2}{*}{ name } & \multicolumn{2}{|c|}{ ellipticity } & \multicolumn{2}{|c|}{ P.A. } & \multirow{2}{*}{$\begin{array}{l}\text { number of } \\
\text { measure - } \\
\text { ments }\end{array}$} & \multirow[t]{2}{*}{ method } & \multirow{2}{*}{$\begin{array}{l}\text { apparent } \\
\text { angular size, } \\
\text { arcsec }\end{array}$} \\
\hline & $\bar{\varepsilon}$ & $\delta \varepsilon$ & $\bar{\theta}$ & $\delta \theta$ & & & \\
\hline G121 & 0.079 & 0.005 & 87 & 11 & 3 & $\mathrm{I}, \mathrm{G}$ & 2.8 \\
\hline G122 & 0.066 & 0.021 & 168 & 12 & 4 & $\mathrm{I}, \mathrm{G}$ & 2.2 \\
\hline G124 & 0.052 & 0.011 & 103 & 1 & 4 & $\mathrm{I}, \mathrm{G}$ & 2.5 \\
\hline G125 & 0.059 & 0.016 & 81 & 10 & 6 & $\mathrm{I}, \mathrm{G}$ & 2.8 \\
\hline G126 & 0.034 & 0.027 & - & - & 1 & G & 2.3 \\
\hline G127 & 0.056 & 0.015 & 162 & 5 & 4 & $\mathrm{I}, \mathrm{G}$ & 3.3 \\
\hline G128 & 0.133 & 0.032 & 55 & 9 & 3 & $\mathrm{I}, \mathrm{G}$ & 4.4 \\
\hline G129 & 0.073 & 0.019 & 58 & 18 & 3 & $\mathrm{I}, \mathrm{G}$ & 2.5 \\
\hline G130 & 0.092 & 0.020 & 114 & 3 & 7 & $\mathrm{I}, \mathrm{G}$ & 3.5 \\
\hline G133 & 0.110 & 0.013 & 46 & 10 & 4 & $\mathrm{I}, \mathrm{G}$ & 2.1 \\
\hline G134 & 0.087 & 0.018 & 48 & 3 & 6 & $\mathrm{I}, \mathrm{G}$ & 3.2 \\
\hline G135 & 0.063 & 0.025 & 114 & 11 & 2 & $\mathrm{I}, \mathrm{G}$ & 2.3 \\
\hline G138 & 0.067 & 0.008 & 110 & 16 & 4 & $\mathrm{I}, \mathrm{G}$ & 2.2 \\
\hline G141 & 0.181 & 0.010 & 178 & 3 & 1 & G & 2.7 \\
\hline G144 & 0.060 & 0.015 & 43 & 6 & 4 & $\mathrm{I}, \mathrm{G}$ & 3.2 \\
\hline G146 & 0.051 & 0.013 & 135 & 7 & 1 & G & 2.4 \\
\hline G147 & 0.072 & 0.006 & 36 & 10 & 3 & $\mathrm{I}, \mathrm{G}$ & 3.6 \\
\hline G148 & 0.099 & 0.029 & 166 & 4 & 9 & $\mathrm{I}, \mathrm{G}$ & 3.7 \\
\hline G150 & 0.181 & 0.010 & 177 & 2 & 4 & $\mathrm{I}, \mathrm{G}$ & 4.2 \\
\hline G151 & 0.040 & 0.006 & - & - & 2 & $\mathrm{I}, \mathrm{G}$ & 1.9 \\
\hline G152 & 0.099 & 0.028 & 14 & 7 & 5 & $\mathrm{I}, \mathrm{G}$ & 2.5 \\
\hline G153 & 0.045 & 0.007 & 147 & 6 & 3 & $\mathrm{I}, \mathrm{G}$ & 2.1 \\
\hline G154 & 0.046 & 0.019 & 142 & 15 & 3 & $\mathrm{I}, \mathrm{G}$ & 3.1 \\
\hline G155 & 0.099 & 0.033 & 41 & 5 & 5 & $\mathrm{I}, \mathrm{G}$ & 2.3 \\
\hline G156 & 0.133 & 0.015 & 10 & 1 & 2 & $\mathrm{I}, \mathrm{G}$ & 3.8 \\
\hline G157 & 0.083 & 0.001 & 117 & 5 & 2 & I & 2.4 \\
\hline G158 & 0.088 & 0.016 & 67 & 9 & 2 & $\mathrm{I}, \mathrm{G}$ & 2.1 \\
\hline G159 & 0.041 & 0.013 & 24 & 5 & 4 & $\mathrm{I}, \mathrm{G}$ & 2.3 \\
\hline G161 & 0.103 & 0.014 & 48 & 7 & 6 & $\mathrm{I}, \mathrm{G}$ & 2.2 \\
\hline G163 & 0.132 & 0.044 & 78 & 8 & 2 & $\mathrm{I}, \mathrm{G}$ & 2.0 \\
\hline G164 & 0.082 & 0.029 & 174 & 4 & 7 & $\mathrm{I}, \mathrm{G}$ & 2.2 \\
\hline G165 & 0.062 & 0.021 & 66 & 6 & 5 & $\mathrm{I}, \mathrm{G}$ & 2.5 \\
\hline G168 & 0.099 & 0.007 & 149 & 11 & 4 & $\mathrm{I}, \mathrm{G}$ & 2.0 \\
\hline G169 & 0.058 & 0.022 & 14 & 7 & 4 & $\mathrm{I}, \mathrm{G}$ & 2.4 \\
\hline G170 & 0.051 & 0.026 & - & - & 1 & G & 1.8 \\
\hline G172 & 0.072 & 0.019 & 71 & 6 & 4 & $\mathrm{I}, \mathrm{G}$ & 3.6 \\
\hline G173 & 0.070 & 0.019 & 26 & 16 & 4 & $\mathrm{I}, \mathrm{G}$ & 2.8 \\
\hline G176 & 0.062 & 0.020 & 32 & 6 & 7 & $\mathrm{I}, \mathrm{G}$ & 3.0 \\
\hline G182 & 0.081 & 0.022 & 33 & 11 & 2 & $\mathrm{I}, \mathrm{G}$ & 1.9 \\
\hline G183 & 0.071 & 0.019 & 53 & 9 & 4 & $\mathrm{I}, \mathrm{G}$ & 3.1 \\
\hline G188 & 0.106 & 0.030 & 60 & 6 & 3 & $\mathrm{I}, \mathrm{G}$ & 3.2 \\
\hline G192 & 0.076 & 0.020 & 48 & 1 & 3 & $\mathrm{I}, \mathrm{G}$ & 2.2 \\
\hline G196 & 0.134 & 0.002 & 38 & 1 & 3 & $\mathrm{I}, \mathrm{G}$ & 2.3 \\
\hline G197 & 0.104 & 0.029 & 54 & 2 & 4 & $\mathrm{I}, \mathrm{G}$ & 2.4 \\
\hline G199 & 0.066 & 0.030 & 38 & 8 & 2 & G & 2.1 \\
\hline G201 & 0.101 & 0.048 & - & - & 1 & G & 1.8 \\
\hline G205 & 0.061 & 0.020 & 165 & 2 & 3 & $\mathrm{I}, \mathrm{G}$ & 4.8 \\
\hline G206 & 0.094 & 0.001 & 119 & 7 & 2 & $\mathrm{I}, \mathrm{G}$ & 1.9 \\
\hline G212 & 0.080 & 0.015 & 76 & 5 & 4 & $\mathrm{I}, \mathrm{G}$ & 2.3 \\
\hline G213 & 0.048 & 0.015 & 99 & 3 & 6 & $\mathrm{I}, \mathrm{G}$ & 4.2 \\
\hline G215 & 0.062 & 0.019 & 24 & 11 & 7 & $\mathrm{I}, \mathrm{G}$ & 2.9 \\
\hline G216 & 0.077 & 0.011 & 59 & 2 & 4 & $\mathrm{I}, \mathrm{G}$ & 2.0 \\
\hline G217 & 0.070 & 0.026 & 52 & 3 & 4 & $\mathrm{I}, \mathrm{G}$ & 3.8 \\
\hline G218 & 0.065 & 0.019 & 65 & 5 & 6 & $\mathrm{I}, \mathrm{G}$ & 2.8 \\
\hline
\end{tabular}


Table 1. continued

\begin{tabular}{|c|c|c|c|c|c|c|c|}
\hline \multirow[t]{2}{*}{ name } & \multicolumn{2}{|c|}{ ellipticity } & \multicolumn{2}{|c|}{ P.A. } & \multirow{2}{*}{$\begin{array}{l}\text { number of } \\
\text { measure - } \\
\text { ments }\end{array}$} & \multirow[t]{2}{*}{ method } & \multirow{2}{*}{$\begin{array}{l}\text { apparent } \\
\text { angular size, } \\
\text { arcsec }\end{array}$} \\
\hline & $\bar{\varepsilon}$ & $\delta \varepsilon$ & $\bar{\theta}$ & $\delta \theta$ & & & \\
\hline G222 & 0.079 & 0.018 & 24 & 2 & 6 & $\mathrm{I}, \mathrm{G}$ & 3.3 \\
\hline G223 & 0.093 & 0.025 & 42 & 8 & 4 & $\mathrm{I}, \mathrm{G}$ & 1.8 \\
\hline G224 & 0.134 & 0.030 & 44 & 1 & 3 & $\mathrm{I}, \mathrm{G}$ & 1.7 \\
\hline G226 & 0.063 & 0.027 & 97 & 9 & 3 & $\mathrm{I}, \mathrm{G}$ & 3.4 \\
\hline G227 & 0.126 & 0.023 & 102 & 3 & 4 & $\mathrm{I}, \mathrm{G}$ & 2.7 \\
\hline G229 & 0.051 & 0.030 & 156 & 8 & 4 & $\mathrm{I}, \mathrm{G}$ & 3.1 \\
\hline G230 & 0.116 & 0.009 & 166 & 1 & 2 & I & 2.5 \\
\hline G231 & 0.089 & 0.017 & 50 & 12 & 5 & $\mathrm{I}, \mathrm{G}$ & 2.2 \\
\hline G232 & 0.072 & 0.004 & 36 & 6 & 1 & $\mathrm{G}$ & 2.0 \\
\hline G233 & 0.142 & 0.013 & 50 & 2 & 7 & $\mathrm{I}, \mathrm{G}$ & 3.8 \\
\hline G234 & 0.159 & 0.012 & 60 & 1 & 4 & $\mathrm{I}, \mathrm{G}$ & 3.5 \\
\hline G235 & 0.102 & 0.011 & 65 & 3 & 6 & $\mathrm{I}, \mathrm{G}$ & 3.4 \\
\hline G239 & 0.080 & 0.017 & 53 & 2 & 4 & $\mathrm{I}, \mathrm{G}$ & 2.5 \\
\hline G241 & 0.101 & 0.024 & 69 & 6 & 4 & $\mathrm{I}, \mathrm{G}$ & 2.4 \\
\hline G243 & 0.093 & 0.025 & 41 & 5 & 5 & $\mathrm{I}, \mathrm{G}$ & 2.4 \\
\hline G244 & 0.051 & 0.014 & 100 & 4 & 2 & $\mathrm{I}, \mathrm{G}$ & 2.4 \\
\hline G250 & 0.080 & 0.004 & 12 & 1 & 3 & $\mathrm{I}, \mathrm{G}$ & 3.3 \\
\hline G252 & 0.064 & 0.020 & 157 & 8 & 4 & $\mathrm{I}, \mathrm{G}$ & 2.2 \\
\hline G254 & 0.048 & 0.006 & - & - & 4 & $\mathrm{I}, \mathrm{G}$ & 2.5 \\
\hline G256 & 0.043 & 0.014 & 15 & 5 & 6 & $\mathrm{I}, \mathrm{G}$ & 3.4 \\
\hline G257 & 0.086 & 0.016 & 24 & 4 & 4 & $\mathrm{I}, \mathrm{G}$ & 3.1 \\
\hline G258 & 0.030 & 0.006 & 32 & 13 & 3 & $\mathrm{I}, \mathrm{G}$ & 2.3 \\
\hline G261 & 0.104 & 0.007 & 51 & 4 & 2 & $\mathrm{I}, \mathrm{G}$ & 2.5 \\
\hline G262 & 0.073 & 0.009 & 42 & 11 & 4 & $\mathrm{I}, \mathrm{G}$ & 2.5 \\
\hline G263 & 0.115 & 0.008 & 94 & 1 & 4 & $\mathrm{I}, \mathrm{G}$ & 4.6 \\
\hline G264 & 0.053 & 0.014 & 58 & 4 & 3 & $\mathrm{I}, \mathrm{G}$ & 2.0 \\
\hline G265 & 0.042 & 0.026 & - & - & 1 & $\mathrm{G}$ & 1.5 \\
\hline G267 & 0.132 & 0.031 & 158 & 3 & 5 & $\mathrm{I}, \mathrm{G}$ & 2.9 \\
\hline G269 & 0.064 & 0.005 & 52 & 4 & 4 & $\mathrm{I}, \mathrm{G}$ & 2.7 \\
\hline G272 & 0.054 & 0.029 & 58 & 3 & 4 & $\mathrm{I}, \mathrm{G}$ & 4.6 \\
\hline G275 & 0.068 & 0.006 & 99 & 3 & 3 & $\mathrm{I}, \mathrm{G}$ & 2.0 \\
\hline G276 & 0.111 & 0.013 & 60 & 3 & 2 & $\mathrm{G}$ & 2.1 \\
\hline G277 & 0.127 & 0.014 & 151 & 3 & 3 & $\mathrm{I}, \mathrm{G}$ & 1.9 \\
\hline G278 & 0.057 & 0.019 & - & - & 1 & $\mathrm{G}$ & 1.9 \\
\hline G279 & 0.112 & 0.022 & 3 & 1 & 3 & $\mathrm{I}, \mathrm{G}$ & 4.1 \\
\hline G280 & 0.026 & 0.011 & 41 & 8 & 3 & $\mathrm{I}, \mathrm{G}$ & 4.0 \\
\hline G281 & 0.068 & 0.015 & 42 & 18 & 4 & $\mathrm{I}, \mathrm{G}$ & 2.0 \\
\hline G282 & 0.036 & 0.025 & 176 & 3 & 3 & $\mathrm{I}, \mathrm{G}$ & 2.9 \\
\hline G283 & 0.068 & 0.008 & 58 & 3 & 2 & $\mathrm{I}, \mathrm{G}$ & 3.8 \\
\hline G284 & 0.038 & 0.008 & 165 & 7 & 2 & $\mathrm{I}, \mathrm{G}$ & 5.0 \\
\hline G286 & 0.094 & 0.010 & 120 & 3 & 4 & $\mathrm{I}, \mathrm{G}$ & 3.8 \\
\hline G287 & 0.099 & 0.017 & 77 & 2 & 5 & $\mathrm{I}, \mathrm{G}$ & 3.5 \\
\hline G290 & 0.053 & 0.031 & - & - & 1 & $\mathrm{G}$ & 2.0 \\
\hline G293 & 0.111 & 0.035 & 41 & 3 & 3 & $\mathrm{I}, \mathrm{G}$ & 2.1 \\
\hline G297 & 0.080 & 0.011 & 48 & 8 & 3 & $\mathrm{I}, \mathrm{G}$ & 1.9 \\
\hline G299 & 0.076 & 0.033 & 34 & 5 & 4 & $\mathrm{I}, \mathrm{G}$ & 2.9 \\
\hline G300 & 0.075 & 0.021 & 163 & 6 & 4 & $\mathrm{I}, \mathrm{G}$ & 5.4 \\
\hline G301 & 0.096 & 0.015 & 41 & 2 & 4 & $\mathrm{I}, \mathrm{G}$ & 2.9 \\
\hline G302 & 0.127 & 0.033 & 80 & 5 & 3 & $\mathrm{I}, \mathrm{G}$ & 4.0 \\
\hline G304 & 0.065 & 0.008 & 160 & 6 & 3 & $\mathrm{I}, \mathrm{G}$ & 5.5 \\
\hline G305 & 0.074 & 0.042 & 19 & 5 & 2 & $\mathrm{I}, \mathrm{G}$ & 3.1 \\
\hline G313 & 0.075 & 0.010 & 145 & 10 & 2 & $\mathrm{I}, \mathrm{G}$ & 2.9 \\
\hline G315 & 0.150 & 0.053 & 106 & 1 & 4 & $\mathrm{I}, \mathrm{G}$ & 4.2 \\
\hline G318 & 0.105 & 0.029 & 47 & 1 & 3 & $\mathrm{I}, \mathrm{G}$ & 3.3 \\
\hline
\end{tabular}


Table 1. continued

\begin{tabular}{|c|c|c|c|c|c|c|c|}
\hline \multirow[t]{2}{*}{ name } & \multicolumn{2}{|c|}{ ellipticity } & \multicolumn{2}{|c|}{ P.A. } & \multirow{2}{*}{$\begin{array}{l}\text { number of } \\
\text { measure - } \\
\text { ments }\end{array}$} & \multirow[t]{2}{*}{ method } & \multirow{2}{*}{$\begin{array}{l}\text { apparent } \\
\text { angular size, } \\
\text { arcsec }\end{array}$} \\
\hline & $\bar{\varepsilon}$ & $\delta \varepsilon$ & $\bar{\theta}$ & $\delta \theta$ & & & \\
\hline G322 & 0.101 & 0.006 & 156 & 3 & 2 & $\mathrm{I}, \mathrm{G}$ & 5.0 \\
\hline G343 & 0.057 & 0.012 & 151 & 7 & 1 & G & 2.6 \\
\hline G348 & 0.081 & 0.005 & 90 & 7 & 1 & I & 3.6 \\
\hline G351 & 0.033 & 0.020 & 14 & 4 & 2 & $\mathrm{I}, \mathrm{G}$ & 5.6 \\
\hline B090 & 0.168 & 0.006 & 64 & 2 & 2 & $\mathrm{I}, \mathrm{G}$ & 1.7 \\
\hline B098 & 0.078 & 0.013 & 56 & 10 & 6 & $\mathrm{I}, \mathrm{G}$ & 4.5 \\
\hline B102 & 0.081 & 0.025 & 70 & 7 & 2 & $\mathrm{I}, \mathrm{G}$ & 2.8 \\
\hline B166 & 0.071 & 0.005 & 46 & 13 & 4 & $\mathrm{I}, \mathrm{G}$ & 2.4 \\
\hline D081 & 0.119 & 0.006 & 64 & 5 & 2 & $\mathrm{I}, \mathrm{G}$ & 2.2 \\
\hline D083 & 0.126 & 0.036 & 74 & 3 & 3 & $\mathrm{I}, \mathrm{G}$ & 2.2 \\
\hline D087 & 0.129 & 0.003 & 74 & 1 & 2 & $\mathrm{I}, \mathrm{G}$ & 2.5 \\
\hline
\end{tabular}

Table 2. Comparison of the ellipticities of GC systems in M 31, Milky Way, and Magellanic Clouds

\begin{tabular}{lcrll}
\hline Galaxy & $\varepsilon \pm \delta \varepsilon$ & sample & interval & source \\
& & & & \\
\hline M 31 & $0.09 \pm 0.04$ & 173 & $0.03 \div 0.24$ & this work \\
M 31 annulus A & $0.08 \pm 0.07$ & 18 & $0.02 \div 0.24$ & Lupton, 1989 \\
M 31 annulus B & $0.12 \pm 0.06$ & 18 & $0.04 \div 0.27$ & Lupton, 1989 \\
M 31 annulus A & $0.06 \pm 0.05$ & 13 & $0.01 \div 0.20$ & Davoust \& Prugniel, 1990 \\
M 31 annulus B & $0.07 \pm 0.05$ & 13 & $0.03 \div 0.20$ & Davoust \& Prugniel, 1990 \\
Milky Way & $0.08 \pm 0.05$ & 93 & $0.00 \div 0.28$ & Frenk \& Fall, 1982 \\
Milky Way & $0.08 \pm 0.06$ & 100 & $0.00 \div 0.24$ & White \& Shawl, 1987 \\
Milky Way & $0.07 \pm 0.04$ & 20 & $0.03 \div 0.30$ & Geyer et al., 1983 \\
LMC & $0.22 \pm 0.07$ & 25 & $0.09 \div 0.33$ & Geisler \& Hodge, 1980 \\
LMC & $0.16 \pm 0.05$ & 49 & $0.06 \div 0.32$ & Kontizas et al., 1989 \\
LMC & $0.13 \pm 0.12$ & 25 & $0.00 \div 0.36$ & Geyer \& Richthler, 1981 \\
LMC & $0.11 \pm 0.07$ & 52 & $0.01 \div 0.33$ & Frenk \& Fall, 1982 \\
SMC & $0.19 \pm 0.06$ & 34 & $0.03 \div 0.33$ & Kontizas et al., 1990 \\
\hline
\end{tabular}

both Lu89 and DP90. In most of the cases the radii measured by us cover A annuli of Lu89 and DP90, and where our radii are larger we compare ellipticities with mean values of both A and B annuli. Our ellipticities are in reasonable agreement with CCD observations of DP90 (marked by crosses in Fig. 7) except for two clusters ( $G 148$ and $G$ 217). Our ellipticities of these two clusters are derived as the mean of four determinations using 3 plates. The mean ellipticities of clusters in common with DP90 differ only within 0.008 , and the mean standard deviation between the individual data is $\sim 0.028$.

The comparison with Lupton's data based on Wilcoxon test shows no significant difference for the sample of 16 common clusters with $95 \%$ confidence level, and an empirical relation $\varepsilon_{\mathrm{Lu} 89}=(1.103 \pm 0.270) \varepsilon_{\mathrm{our}}-$ $(0.003 \pm 0.023)$ is derived. The mean flattening of the two sets are practically the same $(0.080$ and 0.076$)$, but the mean standard deviation between the ellipticities of the individual clusters is $\sigma \approx 0.043$. The maximal absolute deviation for the ellipticities of clusters in common is 0.07 or $\lesssim 2 \sigma$.

Based on six common clusters, Lupton (1989) compares his data with those of our previous works and finds that his values are systematically larger. We do not agree with his opinion that the possible explanation of this discrepancy is the low image quality of our photographic material. The first reason to claim this is that our results are well consistent with CCD observations of DP90. The other reason is that now the number of clusters in common in both samples is more than twice larger thus giving a better basis for comparison. In fact, his ellipticities remain still systematically larger but this discrepancy lies within 
Table 3. Comparison with the data of other authors

\begin{tabular}{|c|c|c|c|c|c|c|}
\hline \multirow[t]{3}{*}{ name } & \multicolumn{5}{|c|}{ ellipticities } & \multirow{3}{*}{$\begin{array}{l}\text { radius } \\
\left({ }^{\prime \prime}\right)\end{array}$} \\
\hline & \multicolumn{2}{|c|}{ DP90 } & \multicolumn{2}{|c|}{ Lu89 } & \multirow[t]{2}{*}{ our data } & \\
\hline & $\mathrm{A}$ & B & $\mathrm{A}$ & $\mathrm{B}$ & & \\
\hline G035 & - & - & 0.07 & 0.15 & 0.07 & 4.00 \\
\hline G052 & - & - & 0.02 & 0.04 & 0.07 & 2.80 \\
\hline G058 & - & - & 0.05 & 0.10 & 0.07 & 6.50 \\
\hline G064 & 0.03 & 0.06 & - & - & 0.04 & 4.70 \\
\hline G070 & - & - & 0.01 & 0.11 & 0.06 & 3.25 \\
\hline G072 & 0.11 & 0.11 & - & - & 0.09 & 2.00 \\
\hline G073 & 0.03 & 0.06 & - & - & 0.05 & 5.00 \\
\hline G076 & 0.05 & 0.03 & - & - & 0.03 & 5.20 \\
\hline G078 & 0.08 & 0.05 & - & - & 0.08 & 6.50 \\
\hline G087 & - & - & 0.04 & 0.12 & 0.04 & 3.30 \\
\hline G096 & - & - & 0.10 & 0.11 & 0.04 & 4.50 \\
\hline G108 & - & - & 0.11 & 0.14 & 0.04 & 3.50 \\
\hline G119 & 0.02 & 0.03 & 0.01 & 0.06 & 0.05 & 5.20 \\
\hline G134 & - & - & 0.06 & 0.09 & 0.09 & 3.20 \\
\hline G148 & 0.05 & 0.07 & - & - & 0.10 & 3.75 \\
\hline G150 & - & - & 0.24 & 0.27 & 0.18 & 4.20 \\
\hline G156 & - & - & 0.13 & 0.19 & 0.13 & 3.80 \\
\hline G213 & 0.05 & 0.07 & - & - & 0.05 & 4.20 \\
\hline G217 & 0.01 & 0.03 & 0.00 & 0.08 & 0.07 & 3.75 \\
\hline G229 & 0.09 & 0.12 & - & - & 0.05 & 3.10 \\
\hline G244 & - & - & 0.06 & 0.09 & 0.05 & 2.40 \\
\hline G280 & 0.02 & 0.03 & 0.07 & 0.04 & 0.03 & 4.00 \\
\hline G302 & - & - & 0.17 & 0.14 & 0.13 & 4.00 \\
\hline G322 & - & - & 0.03 & 0.15 & 0.10 & 5.00 \\
\hline
\end{tabular}

$10 \%$ - a value well below the formal error of the linear fit stated above. The third reason is that our seeing is not $\sim 4^{\prime \prime}$ as Lupton claims. This value is simply the mean radius of the outer isodense traced by us for each cluster of interest (see Table 3, Col. 5). We agree yet with his opinion that the photographic detectors have reached the limit of their resources and it is particularly important for the fainter M 31 clusters. Obviously, more certain data about the morphology of such distant globulars can be provided by CCD observations.

\section{Relations of the ellipticity with other cluster parameters}

Our set of data of the projected ellipticities for a representative sample of globular clusters in M 31 is homogeneous enough and there is a reason to look for some correlations of the ellipticity with other cluster parameters.

\subsection{Ellipticity $\varepsilon-$ Luminosity $M_{0}(V)$}

This correlation is of interest bearing in mind the results of van den Bergh (1983) and Hesser et al. (1984) about the clusters in LMC and NGC 5182 whose ellipticities correlate with their luminosity (the brightest GCs are the flattest ones).

To determine the absolute magnitudes $M_{0}(V)$ of M 31 globulars we have used the visual magnitudes $V$ included in Bologna lists (Battistini et al. 1987). The intrinsic colors of the globulars $(B-V)_{0}$ were obtained using Eq. (4) of Crampton et al. (1985). We have used the distance modulus $(m-M)=24$. 3 taken from Pritchet \& van den Bergh (1987). A subsample was formed containing 164 M 31 globulars with corrected absolute magnitudes $M_{0}(V)$.

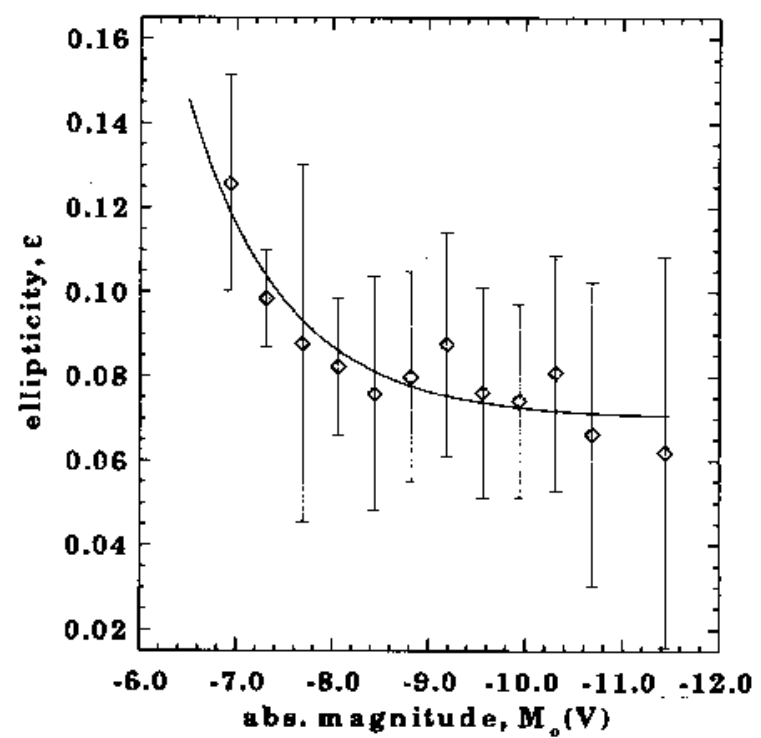

Fig. 8. The projected ellipticities $\varepsilon$ of M 31 globular clusters, plotted against their corrected $M_{0}(V)$ magnitudes. The solid line represents the best fit of the relation $\varepsilon=0.072+50.23 \exp \left(M_{0}(V)\right)$

The projected ellipticities $\varepsilon$ of M 31 globular clusters, derived in our study, as a function of corrected $M_{0}(V)$ magnitudes are plotted in Fig. 8. The ellipticity values were binned in boxes of 0.5 magnitudes and the corresponding standard deviations are also plotted. There is a weak correlation of $\varepsilon$ with $M_{0}(V)$ magnitude in the sense that the brighter clusters are more spherical. The mean absolute magnitude of GCs in our subsample is $\left\langle M_{0}(V)\right\rangle=-8^{\mathrm{m}} \cdot 57 \pm 0.99$. For the clusters brighter than $\left\langle M_{0}(V)\right\rangle$ (77 ones) the mean ellipticity $\bar{\varepsilon}=0.081 \pm 0.033$, and for the fainter clusters (87 ones) $\bar{\varepsilon}=0.086 \pm 0.039$. The Kolmogorov-Smirnov and the Wilcoxon tests do not distinguish these two subdistributions.

Let us define two new subsamples containing the "bright" (brighter than $\left\langle M_{0}(V)\right\rangle-\sigma\left(M_{0}(V)\right)=-9^{\mathrm{m}} \cdot 56$ ) and the "faint" (fainter than $\left\langle M_{0}(V)\right\rangle+\sigma\left(M_{0}(V)\right.$ ) = $\left.-7^{\mathrm{m}} 58\right)$ M31 globulars. When these two ends of the luminosity distribution are compared, the differences between their mean ellipticities become more prominent. For the "bright" GCs (30 ones) $\bar{\varepsilon}=0.075 \pm 0.034$, and 
for the "faint" GCs (26 ones) $\bar{\varepsilon}=0.104 \pm 0.051$. The Kolmogorov-Smirnov and the Wilcoxon tests distinguish these two subsamples with 0.95 and 0.92 confidence.

The error analysis of our processing procedures given above (see Sect. 2.3) shows that such a tendency is physically real. The tendency that the brightest globulars are the roundest ones in M 31 was first mentioned by DP90 on the basis of the ellipticities of 13 M 31 GCs. The analogous tendency for our Galaxy was find by the same authors using the ellipticities of the galactic GCs derived by White $\&$ Shall (1987). There are a few well known exceptions in both galaxies: $\omega$ Cen and M 19 in our Galaxy and Mayall II in M 31 .

\subsection{Ellipticity $\varepsilon-$ Velocity dispersion $\left\langle v^{2}\right\rangle^{1 / 2}$}

In Fig. 9 we compared the velocity dispersions, $\left\langle v^{2}\right\rangle^{1 / 2}$, as derived from integrated light spectra observed at $9 \mathrm{~km} \mathrm{~s}^{-1}$ resolution by Peterson (1989) for 14 M 31 globulars with the ellipticities derived by us.

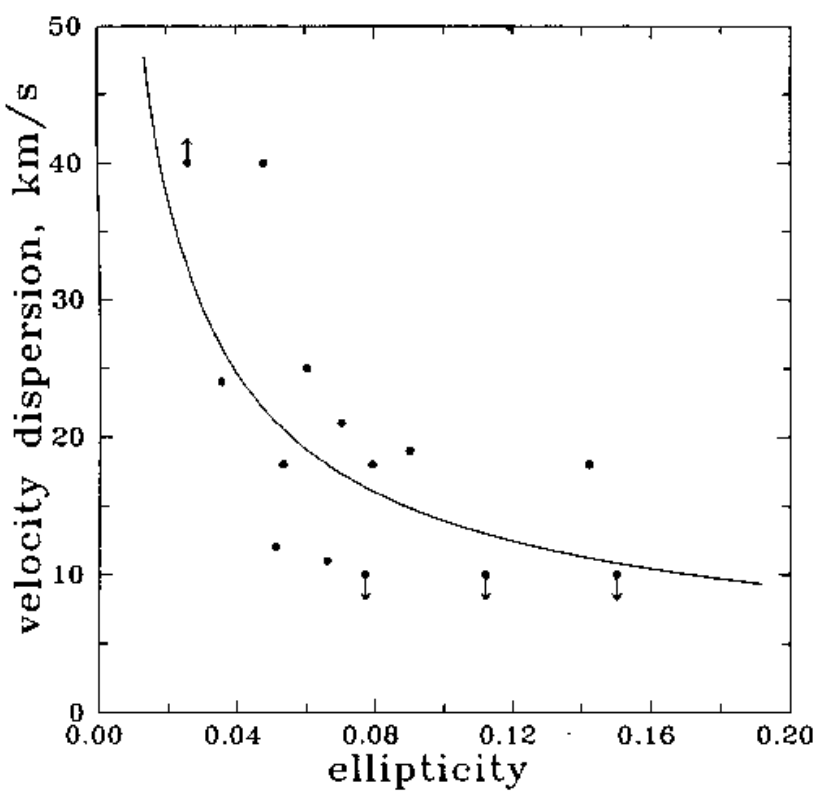

Fig. 9. Velocity dispersions, $\left\langle v^{2}\right\rangle^{1 / 2}$, versus the projected ellipticities $\varepsilon$ for 14 M31 GCs (arrows mark lower and upper limits, respectively). The solid line follows a power-law tendnency $\left\langle v^{2}\right\rangle^{1 / 2} \propto \varepsilon^{-0.6}$

There is a trend the velocity dispersion to take lower values with increasing flattening. This tendency is consistent with the oblate-isotropic model for globular clusters, where the sum of the mean rotation velocity and the mean velocity dispersion, $v_{\text {rot }} /\left\langle v^{2}\right\rangle^{1 / 2}$, is nearly constant to maintain the dynamical equilibrium of the system. Thus the retarding of the rotation (and therefore the decreasing of the $\varepsilon$ ) increases the velocity dispersion $\left\langle v^{2}\right\rangle^{1 / 2}$. More velocity data are needed to investigate this relation. We would like to emphasize that the two relations (Fig. 8 and
Fig. 9) are not independent because of well known FaberJackson relation.

\subsection{Ellipticity $\varepsilon-A g e$}

It is known that in M31 exist massive blue disk clusters and many of them have been identified as globulars. 11 of these constitute a part of our sample. Its intrinsic colors indicate their relative youth $\left(\leq 10^{9} \mathrm{yr}\right.$ ) (Elson \& Walterbos 1988). The derived ellipticities for them show that they are more flattened than the true globulars in M31 (Spassova et al. 1994). The mean ellipticity of the blue clusters is found to be $\bar{\varepsilon}=0.139 \pm 0.044$ compared to $\bar{\varepsilon}=0.086 \pm 0.038$ for a representative sample of other globulars. There is a significant statistical difference between the frequency distribution of the ellipticities of the blue clusters on the one hand and all GCs on the other. According to the evolutionary scenario of Shapiro \& Marchant (1976), this difference may reflect the real difference in the dynamical ages of the two groups of clusters, i.e. the fact that the blue clusters are dynamically younger. Thus the blue clusters may have relaxation times comparable to or longer than their ages, which means that these clusters are not old enough to be relaxed in the usual way.

There is no significant correlation of $\varepsilon$ neither with distance from the center of M 31 nor with the metallicity parameter, $[\mathrm{Fe} / \mathrm{H}]$, from Huchra et al. (1991) and with structural parameters, published by Cohen \& Freeman (1991). Our ellipticity data do not show a tendency for greater flattening of GCs in the direction of the M31 plane and towards its center. Such tendency has been established in our Galaxy by White \& Shawl (1987).

We would like to mention here that the relations discussed above but concerning the true flattening after an appropriate deprojection must be steeper and we plan to investigate this elsewhere.

\subsection{Variations of the ellipticity over the clusters radii}

Some authors (cf. Geyer et al. 1983 and Kontizas et al. 1989) state that the variations of the ellipticity versus the length of major axis (or "radius") within the clusters are a common phenomenon. If the flattening of GCs is due mainly to their rotation, it could be expected that the radial variations of $\varepsilon$ reflect directly over the clusters rotational curve. Pryor et al. (1986) and Meylan \& Mayor (1986) have shown that there is a similarity between the rotational curve and the flattening for M 2 and $\omega$ Cen, respectively. Kontizas et al. $(1989,1990)$ have studied globulars in Magellanic Clouds and have concluded that the inner parts of the clusters in both Clouds are significantly more flattened than their outer parts. An evidence in the opposite sense has been shown by Lupton (1989) for the GCs in M 31: the outer parts of clusters investigated by him seem more flattened than the inner ones. On the other hand, White \& Shawl (1987) have concluded that there 
are no significant variations of $\varepsilon$ with the cluster radial distance for the whole sample of 100 galactic globulars discussed in their work.

In our earlier works (Spassova \& Staneva 1984; Staneva et al. 1985) we have demonstrated some examples for irregular ellipticity variations with radius for a few of the brightest M 31 globulars. However, it is very hard undoubtedly to detect such variations given the scale of the $2 \mathrm{~m} \mathrm{RCC}$ reflector and the seeing conditions at BNAO. Moreover, the isodensity contour analysis, used by us at this time, is quite sensitive to contour deformations caused by the emulsion grains and by the irregular background field at low density levels. Later we reduced the influence due to this analysis, using other processing techniques and stacking the cluster images from a number of plates. Nevertheless, we find no significant evidence that the internal variations of cluster flattening are a phenomenon common to M 31 globulars. In fact, among the 15 brightest clusters there are some objects (e.g. $G 78$ and $G$ 272) where irregular internal variations of $\varepsilon$ within $0.02 \div 0.04$ may be traced. To clear up this question one would have to use the telescopes of new generation like HST.

\section{Conclusions}

We have determined the ellipticities and the orientations of a large sample of 173 globular clusters in Andromeda galaxy. The data considered in this paper suggest some important outdraws about the morphology of the GC population in this galaxy.

1. Our estimations show that most of these clusters are almost spherical ( $70 \%$ of them have $\varepsilon \lesssim, 0.10$ ). The major axes of GCs do not exhibit a preferential orientation toward the M 31 center.

2. The significant statistical difference between the frequency distribution of the ellipticities of the blue clusters in M 31 and all globular clusters, derived by us, confirms the idea of an age dependence, but the number of clusters identified as "young" is very small (only 11 blue clusters versus 162). On the other hand, in the Large Magellanic Cloud where the number of clusters with different age is much greater, there is no compelling evidence for a significant statistical difference among them (Kontizas et al. 1989). Thus the question about the change of the ellipticity with the evolution of the globulars remains still open.

3. We also observe some connection between the ellipticity and the velocity dispersion that follows a power law $\left\langle v^{2}\right\rangle^{1 / 2} \propto \varepsilon^{-0.6}$. Unfortunately, we were not able to substantiate the connections given by our observational uncertainties.

The prediction that $\left\langle v^{2}\right\rangle^{1 / 2}$ of slowly rotating (and less flattened) globulars must be large has been tested by Davoust \& Prugniel (1990), which have examined dynamical consequences of the rotational hypothesis us- ing GC sample in our Galaxy. They have shown that, on the average, there is a tendency the globulars that rotate slower (thus having larger velocity dispersion) to be rounder. On the other hand, White \& Shawl (1987) tested the rotational hypothesis on the basis of other observable effects and showed that in our Galaxy the more concentrated globulars tend to be more spherical.

4. A comparison of the ellipticities of GC populations in some nearby galaxies of Local Group indicates clear morphological differences among them. In M 31, Galaxy - LMC - SMC sequence the leading galaxies (M31 and our one) are spirals of same Hubble type having nearly identical masses. Not surprisingly, the mean ellipticities of GC populations in M31 and in our Galaxy are practically equal, whereas the flattening of M 31 globulars seems to differ markedly from clusters in the LMC and SMC. Probably this is due to much smaller masses of the Magellanic Clouds and to their different evolutionary history.

Obviously, a collection of better-quality morphological data, HST observations for instance, could help to substantiate the picture of the evolutionary status of globular clusters in Andromeda galaxy.

Acknowledgements. We are deeply grateful to the referee, Dr. Philippe Prugniel, for his helpful and constructive comments. Our research was supported by the Bulgarian National Scientific Foundation grant under contract No. F-264/1992 with the Bulgarian Ministry of Education and Sciences.

\section{References}

Battistini P., Bonoli F., Braccesi A., et al., 1980, A\&AS 42, 357 (Bo-Group)

Battistini P., Bonoli F., Braccesi A., et al., 1987, A\&AS 67, 447 (Bo-Group)

Battistini P., Bonoli F., Casavecchia M., et al., 1993, A\&A 272, 77 (Bo-Group)

Bendinelli O., Parmeggiani G., Piccioni A., Zavatti F., 1986, in: The Optimization of the Use of CCD Detectors in Astronomy, ESO/OHP Workshop, OHP, l'Observatoire St. Michel, 17-19 Jun. 1986

Bendinelli O., Parmeggiani G., Piccioni A., Zavatti F., 1987, AJ 94, 1095

Cohen J.G., Freeman K.C., 1991, AJ 101, 483

Crampton D., Cowley A.P., Shade D., Chayer P., 1985, ApJ 288,494

Da Costa G.S., 1992, in: Howell S.B. (ed.) Astronomical CCD Observing and Reduction Techniques. ASP Conf. Ser. 23, 90

Davoust E., Prugniel P., 1990, A\&A 230, 67 (DP90)

Eaton E., 1989, in: Grosb $\phi$ l P. J., Murtagh F., Harris R.H. (eds.), ESO Conf. and Workshop Proc. 31, $1^{\text {st }}$ ESO/ST-ECF Data Analysis Workshop. ESO, Garching bei München, p. 93

Elson R., Walterbos R., 1988, ApJ 333, 594

Frenk C.S., Fall S.M., 1982, MNRAS 199, 565 (FF82)

Geisler D., Hodge P., 1980, ApJ 242, 66 
Geyer E., Richthler T., 1981, in: Davis Philip A.G., Hayes D.S. (eds.) IAU Coll. 68, p. 239

Geyer E., Hopp U., Nelles B., 1983, A\&A 125, 359

Hesser J.E., Harris H.C., van den Bergh S., Harris G.L.H., 1984, ApJ 276, 491

Huchra J.P., Brodie J.P., Kent S.M., 1991, ApJ 370, 495

Janes K.A., Heasley J.N., 1993, PASP 105, 527

Kadla Z.I., Richter N., Strugatskaya A.A., Högner W., 1976, SvA 20, 49

Kadla Z.I., Richter N., Högner W., Strugatskaya A.A., 1977, Izv. Glavnoj Astron. Obs. Pulkovo No. 195

Kontizas E., Dialetis D., Prokakis T., Kontizas M., 1985, A\&A 146, 293

Kontizas E., Kontizas M., Sedmak J., Smaleglia R., 1989, AJ 98, 590

Kontizas E., Kontizas M., Sedmak G., Smareglia R., Dapergolas A., 1990, AJ 100, 425

Kroll P., Neugebauer P., 1993, A\&A (preprint)

Lupton R., 1989, AJ 97, 1350 (Lu89)

Meylan G., Mayor M., 1986, A\&A 166, 122

Pasian F., Santin P., Sedmak G., Smareglia R., 1989, in: Grosb $\phi$ l P.J., Murtagh F., Harris R.H. (eds.) ESO Conf. and Workshop Proc. 31, $1^{\text {st }}$ ESO/ST-ECF Data Analysis Workshop. ESO, Garching bei München, p. 153

Pease F., Shapley H., 1917, Contributions of Mt Wilson Obs. No. 129
Peterson R., 1989, in: Merrit D. (ed.) Dynamics of Dense Stellar Systems. Cambridge Univ. Press, Boston, p. 161

Pritchet C., van den Bergh S., 1984, PASP 96, 804

Pritchet C., van den Bergh S., 1987, ApJ 316, 517

Pryor C., McClure R.D., Fletcher J.M., Hartwick F.D.A., Kormendy J., 1986, AJ 91, 546

Sanders W.L., Schröder R., 1980, A\&A 88, 102

Sargent W.L.W., Kowal C.T., Hartwick F.D.A., van den Bergh S., 1977, AJ 82, 947 (M 31-consortium)

Shapiro S., Marchant A., 1976, ApJ 210, 757

Shapley H., 1930, Star Clusters. McGraw-Hill Publ., New York Spassova N.M., Staneva A.V., 1984, Sov. Astron. Lett. 10, 114 Spassova N.M., Staneva A.V., Golev V.G., 1994, Astrophys. Lett. Comm. (in press)

Spassova N.M., Staneva A.V., Golev V.G., 1988, in: Grindlay J., Philip A.G.D. (eds.) Proc. IAU Symp. 126, Globular Cluster Systems in Galaxies. Reidel, Dordrecht, p. 569

Staneva A.V., Spassova N.M., Baev P.V., 1985, Sov. Astron. Lett. 11, 375

Stetson P.B., 1987, PASP 99, 191

van den Bergh S., 1983, PASP 95, 839

van den Bergh S., Morbey C.L., 1984, ApJ 283, 598

White R.E., Shawl S.J., 1987, ApJ 317, 246 